\title{
DESIGN \& SIMULATION OF LOW LOSS CIRCULAR CORRUGATED WAVEGUIDE FOR 42 GHZ, 200KW GYROTRON
}

\author{
Jinal A. Mistry ${ }^{1}$, S. V. Kulkarni ${ }^{2}$,Shobhit K. Patel $^{3}$, Kunal S. Kadiya ${ }^{4}$ \\ ${ }^{1}$ M.Tech Student, Chandubhai S Patel Institute of Technology, Gujarat, India \\ ${ }^{2}$ Scientist, ICRH, Institute for Plasma Research, Gujarat, India \\ ${ }^{3}$ Assistant Professor, EC Dept., Chandubhai S Patel Institute of Technology, Gujarat, India \\ ${ }^{4}$ Assistant Professor, DT Dept., SMC College of Dairy Science, AAU, Anand, Gujarat, India
}

\begin{abstract}
Corrugated waveguide is used to transmit high power microwave from the microwave devices like Gyrotron. $H E_{11}$ mode has the lowest loss and is a fundamental mode of corrugated waveguide. Corrugated waveguides have proved to be the most efficient waveguides for transmitting high power $\mathrm{GHz} \& \mathrm{THz}$ radiation compared to smooth wall circular waveguide at the operational frequency of the transmission line. This work includes the simulation of circular corrugated waveguide for $42 \mathrm{GHz}$ Gyrotron and also includes the simulation comparison of corrugated metallic waveguide with smooth wall cylinder using HFSS (high frequency structural simulator) software.
\end{abstract}

Keywords: Corrugated waveguide, Gyrotron, $H E_{11}$ hybrid mode, HFSS.

\section{INTRODUCTION}

Transmission of high power microwave radiation is a growing research area due to the increased availability of high power microwave sources. Developing low loss transmission lines is especially important when dealing with high average powers, which can damage components if are not properly designed [1]. Transmission lines are used to transmit power in electromagnetic waves from one point to another. Transmission of high power microwave radiation is a challenging task due to the lossy nature of most fundamental mode transmission lines at such high frequencies. In low-power experiments, simple smooth wall cylindrical waveguides are useful, but when considering the transmission of high power (in MW) for long distances, traditional methods will be insufficient due to large attenuation, mode conversion, beam scattering the possibility of microwave breakdowns, and high heating which can lead to failures in the transmission system. An overmoded approach to transmission lines has been demonstrated as an efficient and practical solution for high power microwave applications [2].

The Gyrotron is a high power microwave tube, which emits coherent radiation at approximately the electron cyclotron frequency or its harmonics. Gyrotron is widely used in plasma fusions, ECRH heating, industrial heating and high temperature processing of materials. Gyrotron oscillators are mainly important in magnetic fusion experiments. Gyrotron technology has significantly advanced towards meeting the goals of providing a reliable and highly efficient source of high-power microwave radiation.

The most common approach is the use of overmoded waveguides, in which the guide radius is much larger than the wavelength. Such waveguides can be corrugated metallic waveguides, dielectric waveguides or dielectric lined metallic waveguides. Free space beaming and corrugated waveguides are closely related as the fundamental HE11 mode used in corrugated waveguides has a conversion efficiency of $98 \%$ to the Gaussian beam which is used in quasi-optical transmission [1]. Overmoded metallic circumferentially corrugated cylindrical waveguides have been shown to have low attenuation over long distances, but may result in high amounts of mode conversion and large losses due to misalignment or manufacturing errors. Decreasing the attenuation and mode conversion in high power experiments is necessary for the integrity of the transmission system as well as the success of the experiment, particularly when transmission over long distances is necessary [2].

\subsection{Need of Corrugated Waveguide}

Following are the advantages of the HE11 mode through a circular corrugated waveguide:

1) Efficient plasma heating requires axis-symmetric narrow pencil beam with well-defined polarization incident on the plasma, the almost perfectly linearly polarized $\mathrm{HE}_{11}$ hybrid mode satisfy these condition best.

2) Low transmission loss \& Large bandwidth in $\mathrm{HE}_{11}$

3) Mode conversion loss at miter bends for $\mathrm{HE}_{11}$ is only about $35 \%$ to that for $\mathrm{TE}_{01}$. The $\mathrm{HE}_{11}$ mode is better separated from competing modes, so propagation is less sensitive to wall distortions [3]. 
4) The $\mathrm{HE}_{11}$ mode at open-ended corrugated waveguide couples directly to the fundamental free space Gaussian mode with less than $2 \%$ loss over wide bandwidths [3].

5) $\mathrm{HE}_{11}$ mode, the fundamental mode for corrugated waveguides, has power concentrated in the center and small fields at the walls of the waveguide, whereas $\mathrm{TE}_{01}$ and $\mathrm{TM}_{11}$ have the lowest loss modes for smooth wall waveguides and have power that is off-center and more susceptible to losses due to fields present at the walls [2].

6) For oversized smooth-wall circular waveguide, the lowest loss mode is $\mathrm{TE}_{01}$, which is not the fundamental mode. This property leads to the danger of mode conversion to lower order modes that are hard to filter out. Also, the $\mathrm{TE}_{01}$ mode is degenerative with the $\mathrm{TM}_{11}$ mode, increasing the possibility of mode conversion to an undesirable mode. On the other hand, corrugated cylindrical waveguides have the lowest loss in the fundamental $\mathrm{HE}_{11}$ mode, reducing the concerns for mode conversion to degenerate with lower order modes [2]. Corrugated waveguides have been used in a variety of applications for the transmission of $\mathrm{GHz}$ radiation for spectroscopic and plasma heating experiments.

\subsection{Brief Introduction of Corrugated Waveguide}

\section{for $42 \mathrm{GHz}, 200 \mathrm{kw}$ Gyrotron}

The gyrotron has become an integral part of the fusion reactors for pre-ionization, current start-up, heating, current drive and for stabilizing neo-classical tearing modes etc. The required frequency of gyrotron lies in the range of $42 \mathrm{GHz}$ to $170 \mathrm{GHz}$ and depends upon the toroidal magnetic field of the tokamak. The power requirement also varies according to tokamak and final requirement is from $5 \mathrm{MW}$ to $20 \mathrm{MW}$. The five organizations of India are developing $42 \mathrm{GHz}, 200$ $\mathrm{kW}$ gyrotron as a part of indigenous development and IPR is involved with the project with major responsibilities as well is the end user of the gyrotron for doing physics experiments on tokamaks ADITYA and SST-1.

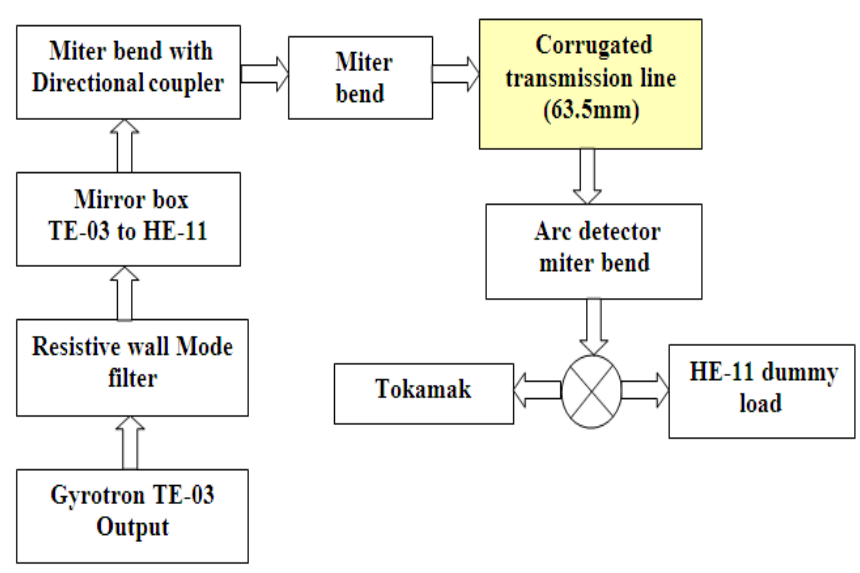

Fig-1: Block diagram of design flow from gyrotron output to the Tokamak / dummy load
Fig. 1 shows the conversion of TE-03 mode to HE-11 mode and then transmission of microwave power from gyrotron to either tokamak or HE-11 dummy load. In this work we present the design \& simulation of circular corrugated waveguide of $63.5 \mathrm{~mm}$ diameter for $42 \mathrm{GHz}, 200 \mathrm{~kW}$ gyrotron. The diameter $63.5 \mathrm{~mm}$ is chosen to make it compatible with inner diameter of miter bend.

\section{DESIGN OF HIGH POWER LOW LOSS CIRCULAR CORRUGATED WAVEGUIDE}

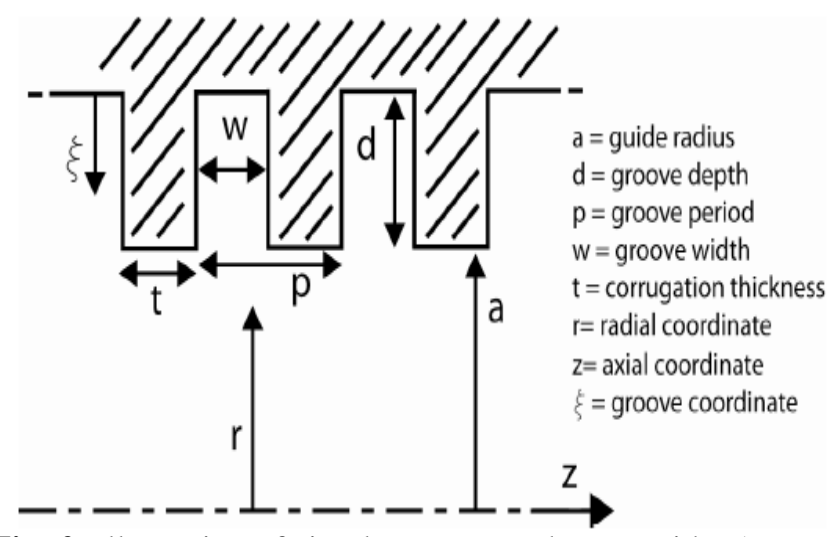

Fig -2: Illustration of circular corrugated waveguide [1]

Fig. 2 shows a schematic of a circular corrugated waveguide with rectangular grooves. To design corrugated waveguide for low losses, the radius $a$ should be much larger than a wavelength $\lambda$, the corrugations are optimized when the groove depth $d \approx \lambda / 4$, period $p \approx \lambda / 3$ and groove width $w<p / 2$. The corrugation tooth thickness is denoted by $t=p-w[1]$. Using above criteria we have calculated the design parameters for the circular corrugated waveguide and are given in Table 1.

Table 1: Calculated Design parameters of circular corrugated waveguide

\begin{tabular}{|c|c|c|}
\hline $\begin{array}{c}\text { Sr. } \\
\text { No. }\end{array}$ & Parameter & Value \\
\hline 1 & Operating frequency (f) & $42 \mathrm{GHz}$ \\
\hline 2 & Wavelength ( $\lambda$ ) & $7.14 \mathrm{~mm}$ \\
\hline 3 & Radius (a) & $31.75 \mathrm{~mm}$ \\
\hline 4 & Corrugation depth (d) & $1.785 \mathrm{~mm}$ \\
\hline 5 & Corrugation periodicity (p) & $2.37 \mathrm{~mm}$ \\
\hline 6 & Corrugation thickness (t) & $1.37 \mathrm{~mm}$ \\
\hline 7 & Groove width (w) & $1 \mathrm{~mm}$ \\
\hline 8 & Waveguide material & $\begin{array}{c}\text { Aluminum , copper } \\
\text { stainless steel }\end{array}$ \\
\hline
\end{tabular}




\subsection{Simulation of Corrugated Waveguide in HFSS}

In this work, low loss circular corrugated waveguide is designed for $42 \mathrm{GHz}$ Gyrotron source using above design parameter in HFSS. And this project also shows the comparison between smooth wall circular waveguide and circular corrugated waveguide and analyzed their Sparameters using HFSS.
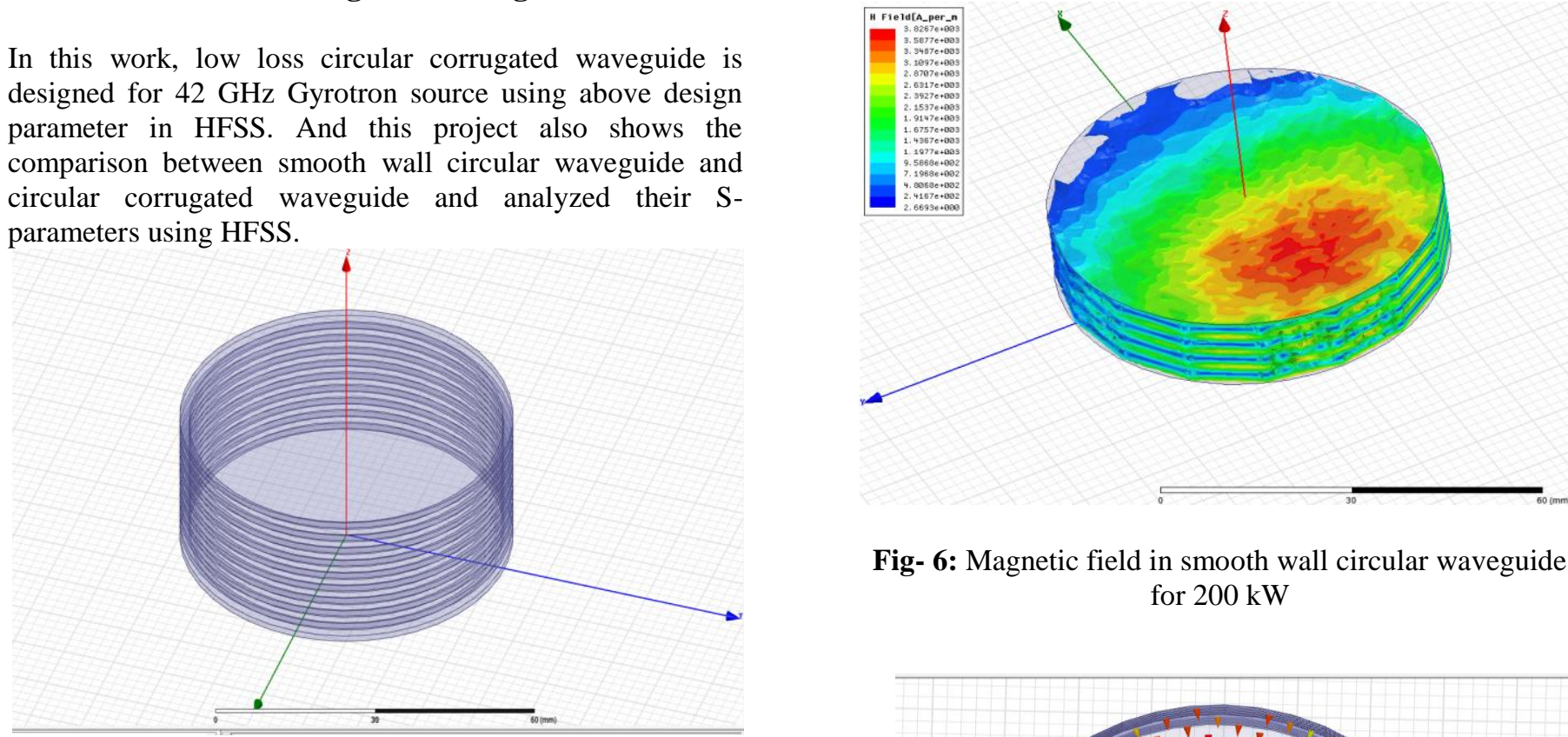

Fig- 6: Magnetic field in smooth wall circular waveguide for $200 \mathrm{~kW}$

Fig-3: Corrugated circular waveguide in HFSS (all dimensions according to Table 1)

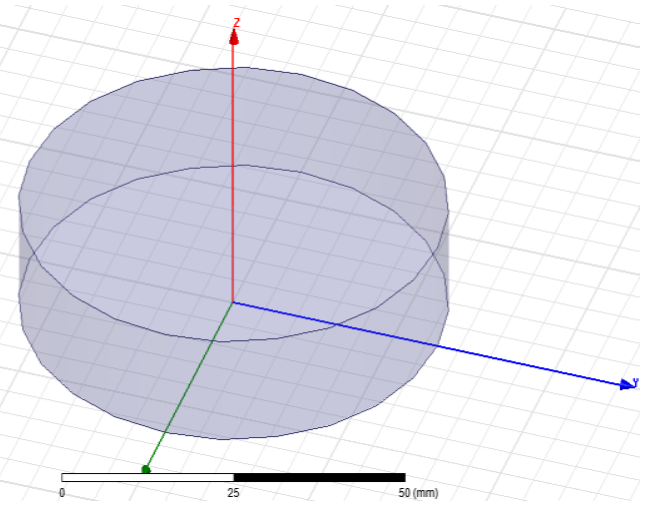

Fig-4: Smooth wall circular waveguide in HFSS

\subsection{Simulation Results from HFSS \& Discussions}
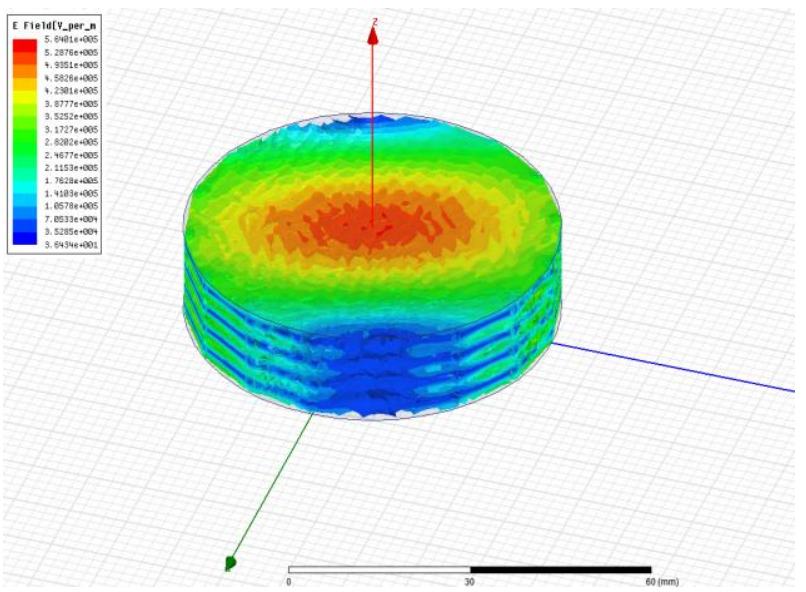

Fig- 5: Electric field in smooth wall circular waveguide for $200 \mathrm{~kW}$
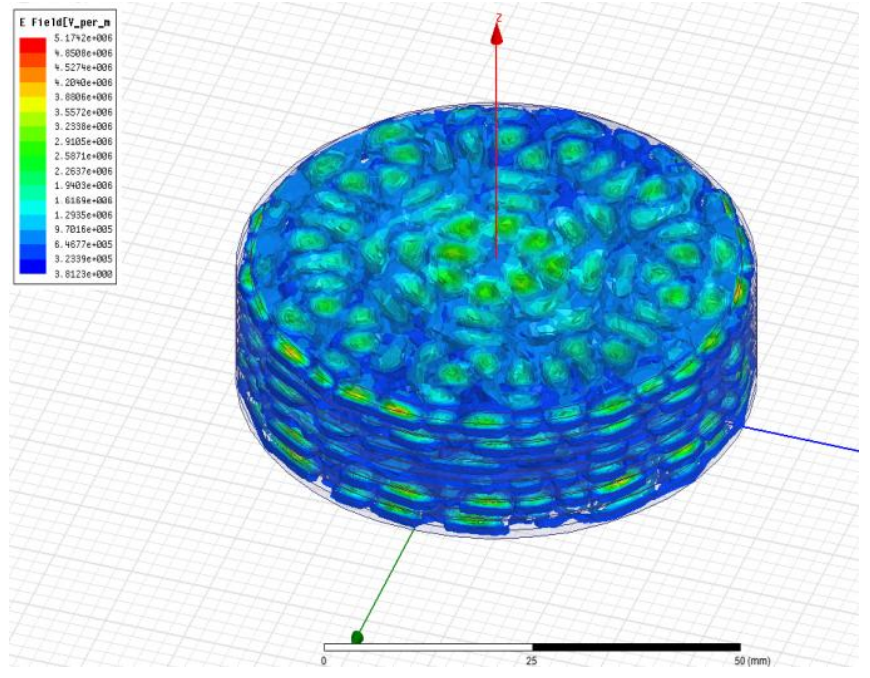

Fig- 8: Electric field in circular corrugated waveguide for $200 \mathrm{~kW}$ 


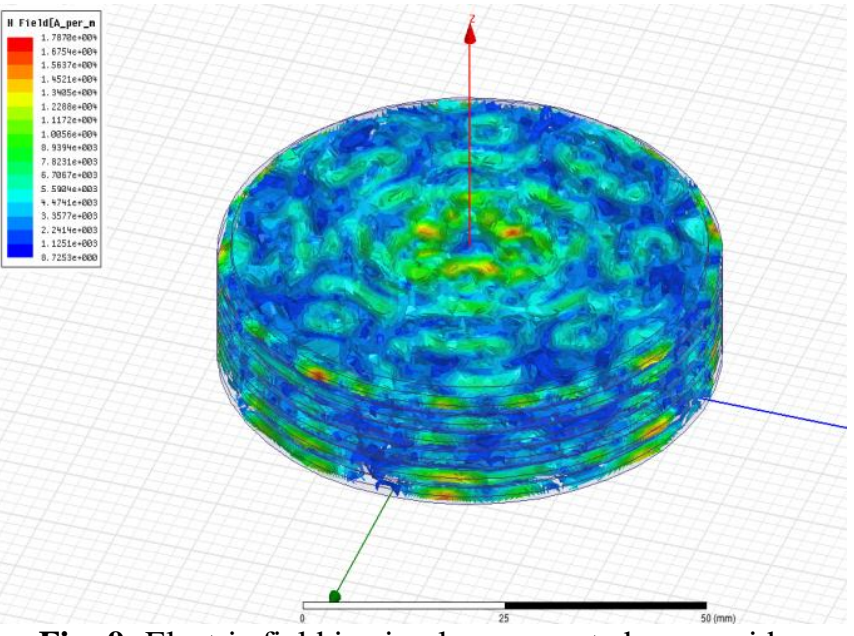

Fig- 9: Electric field in circular corrugated waveguide for $200 \mathrm{~kW}$

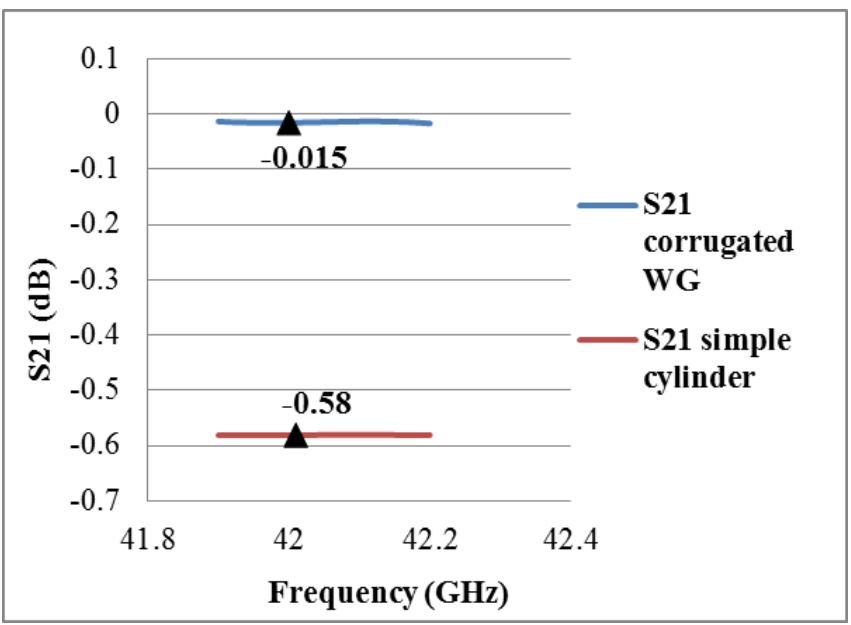

Fig-10: Comparison of Insertion loss (S21) between corrugated waveguide and smooth wall waveguide

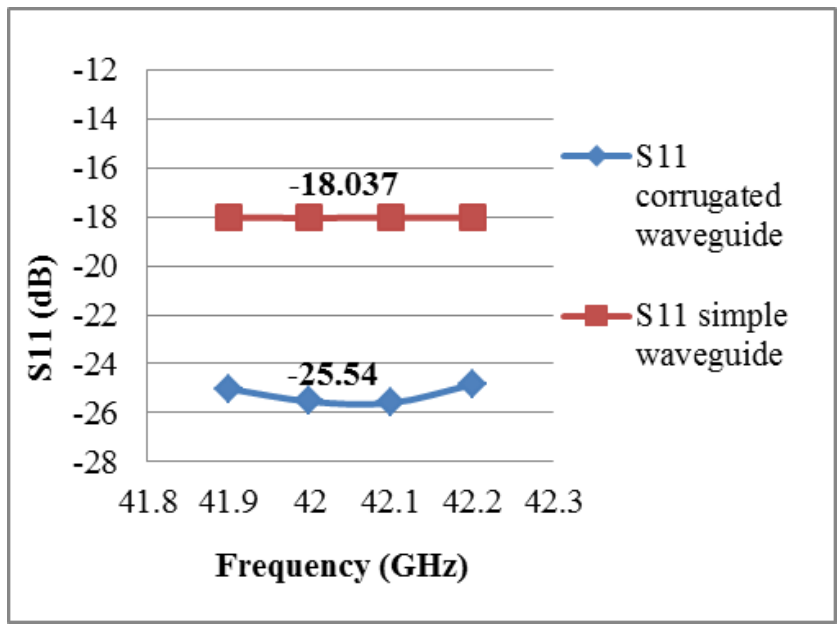

Fig-11: Comparison of Return loss (S21) between corrugated waveguide and smooth wall waveguide
Figures 5 to 9 show the magnitude of electric \& magnetic field in smooth circular waveguide and corrugated waveguide, electric \& magnetic field distibution in smooth circular waveguide and corrugated waveguide and also shows the fundamental mode HE11. The electric \& magnetic field in circular corrugated waveguide is higher than the smooth wall circular waveguide which clearly shown in the above figures.

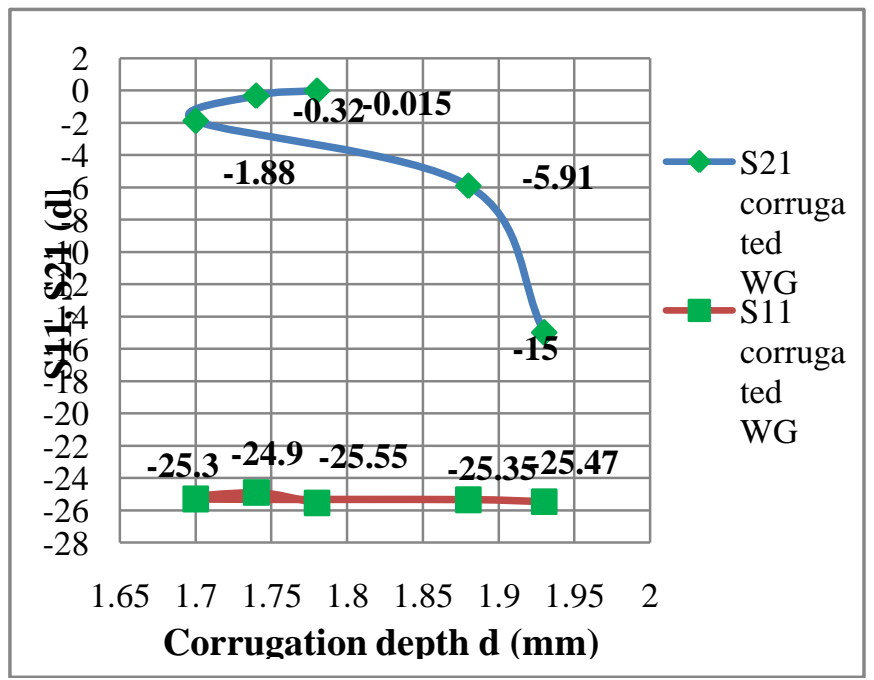

Fig-13: Variations of S11 and S21 parameters with corrugation depth (d) of circular corrugated Waveguide

Fig. 10 and 11 shows the comparison between circular corrugated waveguide and simple circular waveguide at $42 \mathrm{GHz}$ frequency, which shows that the insertion and return losses are less at such high frequency in corrugated waveguide compared to simple circular waveguide. If corrugation depth (d) is varied from its optimized depth $(\approx \lambda / 4)$ then insertion loss (S21) is increased but very little change in the return loss (S11) which is shown in the fig. 13, also it shows that insertion loss is more depends on corrugation depth $(\mathrm{d})$.

Table 2: Results of variation in material used for the corrugated waveguide in HFSS

\begin{tabular}{|c|c|c|c|}
\hline $\begin{array}{c}\text { Sr. } \\
\text { No. }\end{array}$ & $\begin{array}{c}\text { Variation in } \\
\text { Material used for } \\
\text { corrugated } \\
\text { waveguide }\end{array}$ & $\begin{array}{c}\text { Insertion } \\
\text { loss S21 } \\
(\mathrm{dB})\end{array}$ & $\begin{array}{c}\text { Return loss } \\
\text { S11 } \\
(\mathrm{dB})\end{array}$ \\
\hline 1 & Copper & -0.014 & -25.56 \\
\hline 2 & Aluminum & -0.015 & -25.53 \\
\hline 3 & Stainless steel & -0.044 & -24.73 \\
\hline
\end{tabular}


Although the conductivity of copper is greater than aluminum, the insertion losses for aluminum is almost same as copper (shown in Table 2). We have also calculated the change in temperature $\Delta \mathrm{T}$ by passing $200 \mathrm{~kW}$ for $1 \mathrm{sec}$ duration for $\mathrm{Cu}$ and $\mathrm{Al}$ by using equation

$$
\mathbf{Q}=\mathbf{m C} \Delta \mathbf{T}
$$

Where $\mathrm{m}$ is mass of sample, $\mathrm{C}$ is specific heat capacity and $\Delta \mathrm{T}$ is the difference between initial temperature and temperature after $1 \mathrm{sec}$ duration and found it as $0.00127^{\circ} \mathrm{C}$ for copper and $0.00178{ }^{\circ} \mathrm{C}$ for aluminum which is almost same. Based on additional advantage of mass $(101.55 \mathrm{gm}$ for $\mathrm{Al}, 337 \mathrm{gm}$ for $\mathrm{Cu}$ ) and also due to its low cost and less weight we preferred aluminum to be used in our design.

Table 3: Summary of design Results (at $42 \mathrm{GHz}$ ) in HFSS

\begin{tabular}{|c|c|c|c|}
\hline $\begin{array}{c}\text { Sr. } \\
\text { No. }\end{array}$ & $\begin{array}{c}\text { Results } \\
\text { parameters }\end{array}$ & $\begin{array}{c}\text { Smooth wall } \\
\text { cylinder }\end{array}$ & $\begin{array}{c}\text { Corrugated } \\
\text { waveguide }\end{array}$ \\
\hline 1 & $\begin{array}{c}\text { Insertion loss } \\
\text { S21(dB) }\end{array}$ & -0.58 & -0.015 \\
\hline 2 & $\begin{array}{c}\text { Return loss } \\
\text { S11 } \\
(\mathrm{dB})\end{array}$ & -18.04 & -25.55 \\
\hline 3 & VSWR & 1.3 & 1.1 \\
\hline
\end{tabular}

\section{CONCLUSIONS}

This work has presented the design and simulation of high power low loss circular corrugated metallic waveguide for $42 \mathrm{GHz}$ Gyrotron. And also presented the simulation comparison between smooth wall circular metallic waveguide and corrugated circular metallic waveguide. Simulation results shows that the corrugated waveguide have low insertion and return loss compared to smooth wall circular waveguide. Insertion loss is affected by varying the corrugation depth (d), whereas return loss is not that much dependent on corrugation depth (d). For microwave transmission, material choice is also important for design.

Corrugated waveguides have proved to be a reliable technique with regards to their low loss nature and ability to handle high powers with low losses.

\section{ACKNOWLEDGMENT}

This work was carried out at Institute for plasma research, Gandhinagar, India. The authors are thankful to the Director and ICRH group of Institute for plasma research, Gandhinagar for all their support and guidance.

\section{REFERENCES}

[1] Nanni E.A., Jawla S. K., Shapiro M. A., Woskov P. P., Temkin R. J., "Low Loss Transmission Lines for High Power Terahertz Radiation", Plasma Science and Fusion Center, Massachusetts Institute of Technology, Cambridge, MA, 02139 USA

[2] Elizabeth J. Kowalski, thesis on "Miter Bend Loss and Higher Order Mode Content Measurements in Overmoded Millimeter-Wave Transmission Lines", Submitted to the Massachusetts institute of technology, Cambridge, MA 02139, United States, September 2010

[3] http://www.ga.com/straight-corrugated-waveguides

[4] Ansys HFSS Version 15.0 software 